\title{
Two-dimensional Verticalcavity Surface-emitting Laser Arrays Fabricated by Zinc Diffusion
}

\author{
J. L. Yen, and Y. J. Yang \\ Department of Electrical Engineering, National Taiwan University, Taipei, Taiwan \\ C. Y. Lin \\ Analytical Instrumentation Center, National Taiwan University, Taipei, Taiwan
}

\begin{abstract}
Vertical-cavity surface-emitting laser (VCSEL) arrays with seven devices arranged in a honeycomb pattern have been fabricated by a selective zinc diffusion process. An optical coupled theory is used to predict the mode behavior, it has been found that a mixing in-phase, out-phase supermodes, and individual single mode operation has been observed and the power ratio among these modes strongly dependents on the device spacing.
\end{abstract}

Vertical-cavity surface-emitting bsers (VCSELs) array is one of the alternatives for getting a large coherent output power due to its feasibility of making a large scale 2-D device. Most reported VCSEL arrays operate in out-phase supermode, or mixing modes. One coherent in-phase antiguided VCSEL array is demonstrated. ${ }^{[1]}$ In our experiment, we present a VCSEL array that can be fabricated by a simple zinc diffusion process. ${ }^{[2]}$ Disordered distributed Bragg reflectors (DBRs) are selectively formed in-between the elements by zinc diffusion in order to suppress the out-of-phase array supermode which exhibit an intensity peak in the spacing region. An optical coupled mode theory is also used to predict the mode behavior and calculate the power ratio among the modes. ${ }^{[3]}$ The VCSEL array consists of seven 5 um elements with spacing varied with 3.5 um, $6 \mathrm{um}, 8.5 \mathrm{um}, 11 \mathrm{um}$, and $13.5 \mathrm{um}$ to investigate the distance dependence on the mode coupling. For the in-phase case, most power concentrates at the center lobe, while for the out-phase case, the most power splits into two symmetrical side lobes. The measured far field data on the fabricated devices shows that the array emits light in the center as well as the two symmetrical off-axis directions. It indicates the array lases at both in -phase and out-of-phase supermodes. By fitting the measured far field intensity to those of simulated in-phase, out-of-phase supermodes and individual single mode, we can find the power ratio of each mode. The results of VCSEL array with 3.5 um and 6 um spacing are shown in Fig. 1 and Fig. 2 respectively, in which it shows that about $20-30 \%$ total power of in -phase mode can be achieved in this configuration. For the array with spacing larger than $6 \mathrm{um}$ most arrays exhibit individual single mode operation, in which all the elements lase individually at single mode but incoherently one another. These results indicate that the disordered DBR with spacing larger than 6 um provides a high optical loss region to effectively suppress the higher-order mode to form an individual single mode operation. The coupling efficiency between each element will become stronger when the spacing or disorder region gets narrower, which will start to favor out-of-phase mode emission resulting in a mixture mode operation, occurred in the array with spacing smaller than $6 \mathrm{um}$.

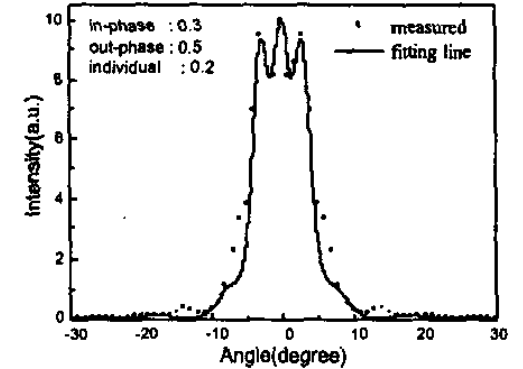

Fig. 1. The measured far field intensity of a VCSEL array with $3.5 \mu \mathrm{m}$ spacing.

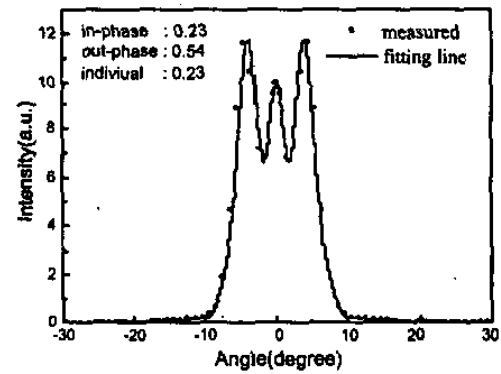

Fig. 2. The measured far field intensity of a VCSEL array with $6 \mu \mathrm{m}$ spacing.

\section{References}

[1] D. Zhou and L. J. Mawst, "Two-dimensional phase-locked antiguided vertical-cavity surface emitting laser arrays," Appl. Phys. Lett., Vol. 77, pp. 2307-2309, (2000).

[2] C. C. Chen, S. J. Liaw, and Y. J. Yang, "Stable single-mode operation of an 850-nm VCSEL with a higher order mode absorber formed by shallow Zn diffusion," IEEE Photon. Technol. Lett., Vol. 13, pp. 266-268, (2001).

[3] P. K. Cheo, A. liu, G. G. King, "A high-brightness beam from a phase-locked multicore Yb-doped fiber laser array," IEEE Photon. Technol. Lett. Vl. 13, pp. 439-441, (2001). 